\title{
SEC's Electronic Filing System: An Evaluation
}

Dr. David C. Yang, Accountancy, University of Hawaii at Manoa

\begin{abstract}
This paper discusses the development and functioning of the SEC's EDGAR system. The considerations for using EDGAR and the future impact of such a revolutionary system on accounting issues and practices such as continuous reporting, continuous auditing and database auditing, etc. are examined.
\end{abstract}

\section{Introduction}

Many aspects of today's business practices have changed dramatically. The advancement in computer technology, particularly in the area of microcomputers, telecommunication, and database has enabled many companies today to use computer technology to their best advantage. Companies use information technology in many areas to make their businesses run more efficiently and effectively. The function of accounting in a company is usually the first place where computerization takes place. Accounting data has been computer-processed for internal purposes day-to-day operations or management decisions. The use of computer technology for external reporting purposes has just begun to receive more attention now. The Security and Exchange Commission (SEC) has taken the first step towards the development of an electronic data filing and retrieval system called EDGAR. It offers an opportunity to affect a dramatic change in the way financial reports are filed and delivered. It poses certain considerations for people involved, namely, the SEC, the filing corporations, the investing public, the financial analysts and the accounting professionals. The idea of EDGAR is a revolutionary one and its impact is bound to be tremendous.

This paper will discuss the development and functioning of the EDGAR system. Also, considerations for using EDGAR and the future impact of such a revolutionary system on accounting issues and practices will be discussed.

\section{Development of EDGAR}

EDGAR stands for Electronic Data Gathering Analysis and Retrieval. This system allows electronic filing, processing, and dissemination of companies' financial information. The development of EDGAR is to fulfil one of the major objectives of SEC. During the last fifty years of its existence, the SEC has promulgated and administered disclosure rules based on the premise that an informed public receiving timely and complete information is adequately equipped to invest in a wise manner. As a result, one of the major accounting functions of the publicly owned companies is to provide financial statements that have been prepared by management and examined by independent auditors. For many years, printed reports are required by the SEC. These reports are received by the Commission, processed, and made available to the general public. Since the number of companies filing with the SEC have increased so rapidly, the SEC has been faced with the problem of maintaining its quality and efficiency in the future.

To keep pace with the continuously growing workload, the SEC began to rely on electronic data processing equipment in the 1970's to track 
the physical flows of reports filed by corporations. However, the reports received have been on paper. The Commission realized the problem that a technological gap was created between a disclosure system based solely on paper and a business environment that has been computerized to a large extent.

A staff task force was formed by the SEC in 1983 to solicit comments on the feasibility of developing an electronic disclosure system that would be able to receive reports electronically, allow electronic reviews by the staff and provide computerized dissemination to users. This was the first attempt by the SEC to make use of the current information technology in the areas of electronic communication and database concept. If this system becomes feasible, the SEC will be able to accomplish its objective more effectively with the help of computer technology. Availability of financial information to investing public will be virtually instantaneous.

In May 1984, the SEC granted a contract for developing a pilot system to test the feasibility of such an information gathering and dissemination system. By September the same year, the SEC received registration statements and periodic reports electronically from volunteer filers. Companies filing under the Public Utilities Holding Company Act have been using EDGAR since July 1985. In November 1985, a large number of investment companies and unit investment trusts participated in the EDGAR system. Over 10,000 electronic filings have been received since September 1984.

\section{How EDGAR Works}

Basically there are three phases in the functioning of the EDGAR system, namely, submission of reports, processing of filings, and dissemination of reports.

\section{A. Submission of Reports}

EDGAR was designed to allow great flexibility in the ability of the filers. The SEC currently allows the registrants to submit their filings in any of the three different media - magnetic tape, diskette, or direct telephonic transmission. The third method is much more advantageous than physical delivery of tapes or diskettes, especially for those companies which file frequent registration statements or update prospectuses. The purpose of allowing different media is to make it easier for the companies to use their existing hardware equipment.

Under EDGAR, there are no "standard forms" required to present and submit financial reports. Except for some submission header information, filers are free to report in any convenient manner.

\section{B. Processing of Filings}

Electronic filings are processed at the Commission by pilot branches in the Corporate Finance Division and the Investment Management Division as well as by the office of Public Utility Regulation. Experienced accountants, attorneys, and financial analysts volunteer to work in the pilot branches. The same selective review criteria applied to paper filings are applied to electronic filings. The difference is that the review process is faster and more efficiently carried out at the computer work stations which allow the use of computerized spreadsheet, word processor, and database inquiry technology to complete the examination. Comments on the report are electronically assembled into the comment letter which can be electronically transmitted to the registrants when the staff's work is completed.

\section{Dissemination of Reports}

After the filings have been received and accepted, they are immediately available throughout the Commission and in any of three Public Reference Rooms (New York, Washington, and Chicago). The computer generated microfiche is prepared for the day's filings each night. The preparation of this microfiche is about two weeks faster than conventional paper filings. 


\section{Benefits and Drawbacks of EDGAR}

The EDGAR system benefits not only the SEC but also the filing corporations and the investing public. Benefits received by each of these groups and drawbacks will be discussed in this section.

\section{A. Benefits to the SEC}

The SEC anticipates improvements in the areas of storage space, information retrieval, and the process of reviewing filings. Currently, the SEC has to handle the equivalent of over seven million pages of documents each year (1986, Liebtag). The EDGAR system facilitates the receipts, cataloging, and the storage of filings. In the long run, this will help the commission save more money. Moreover, filing reviewers of the SEC can monitor filings from their computerized workstations if the filings are directly transmitted over the telephone line. The information received can be summarized and analyzed using spreadsheet and other software packages. These capabilities help speed up the review process.

\section{B. Benefits to Filing Corporations}

The main advantage for the corporations is the speed of submission. Organizations that submit their filings through EDGAR can maintain physical control of documents till the last minute before the deadline. Physical delivery of filings takes much longer to file. Another important advantage is that the companies can have their reports or prospectuses processed much faster than before. The process of receiving and responding to a comment letter from the SEC reviewer which could take more than a week can now be done in just a day. With this speedy process, corporations can have faster access to the security market.

\section{Benefits to Investors}

When the EDGAR system is fully operational, investors will be able to receive filed information virtually instantaneously through an information service. Presently, EDGAR is still in its pilot stage. The general public can access the corporate filings through microcomputers set up in the SEC's public reference rooms in Chicago, New York, and Washington D.C.

\section{Drawbacks of EDGAR}

Even though EDGAR was designed to facilitate financial analysis, there are some drawbacks at this pilot stage of the system. Certain incremental costs were incurred to implement EDGAR, however, initial study showed that these costs were not very significant for pilot participants (see Arnold and Diamond, 1986). Since EDGAR filings are submitted in free text form, it is currently not possible to select individual data items for analysis without manual intervention. Searching for individual items on the text is not very efficient at this time. Multiple location of certain analytical terms and phrases that may appear in the text makes the searching process not as efficient as the users prefer.

\section{Considerations for Using EDGAR}

The use of EDGAR for filing financial reports offers a number of benefits to the companies. In order to take full advantage of the EDGAR, companies filing with SEC should consider certain factors when using EDGAR. The most common considerations are internal control for using EDGAR and the medium used for filing.

\section{A. Internal Control}

Filing through EDGAR involves the use of electronic equipment and procedures. Therefore, certain EDP internal controls must be designed to prevent problems.

Filing by direct transmission requires the use of PINs (Personal Identification Numbers) instead of signatures for paper filings. There has been a controversy about the use of PINs. Companies feel uncomfortable to use PINs. Some of them express that one could use another's PIN to affect filings that are not authorized by the company. However, in order to gain access to affect the 
electronic filing, the person also has to know the company's password - which is another security identifier given by the SEC. To control unauthorized use of PINs and passwords, companies should have proper organization control (more preventive in nature) that ensures that only authorized personnel know the PINs and passwords. Also, control over unauthorized access to computer equipment, software programs, and data files can help minimize the risk of unauthorized alteration of filings.

\section{B. Medium for filing}

EDGAR allows any one of the three media (tape, diskette and direct telephonic transmission) for filings. Companies should determine the medium to be used in consideration of their needs, costs, and the advantages offered by each medium. For example, a company that has frequent filings with SEC and desires to have the fastest filing and review process would prefer the direct telephonic transmission. In choosing the use of direct transmission, the company should also consider the two principle ways to accomplish the filings through EDGAR. These two ways of transmission are: (1) directly from the company's office or (2) through financial printers who are cooperating with the SEC in the development of this project. Most companies choose to file from their own offices due to the concern about the security of passwords.

In addition, in choosing the medium for filing, companies should consider the availability of electronic equipment. For direct transmission, a company can use a microcomputer and a modem to accomplish the job. Mainframe equipment with necessary data transmission capabilities can do the job as well.

\section{Future Implications of EDGAR}

Even though the EDGAR system is still considered to be in its infancy, it is a big step towards the future trend in financial reporting. There are certain perceived implications which go beyond the benefits and problems that we can see today about EDGAR. Some of them that will be discussed here are:
A. Continuous reporting
B. Continuous auditing
C. Database auditing
D. Efficient and relevant financial analysis
E. Amount of information reported
F. References for CPAs

\section{A. Continuous Reporting}

Some people see the development of EDGAR as the first step towards the possibility of continuous reporting in the future (Yang, 1987). It is not surprising that the objective of the SEC to provide more timely and relevant information to investing public and the rapid development of information technology may force continuous reporting as a possible requirement in the future.

Some knowledgeable professionals have predicted that continuous reporting is not too far from now (Simon, 1985; Goodfellow, 1985). According to Burton (Smith, 1984, p. 73), in ten to fifteen years, corporate financial statements will be supplanted by a steady flow of computer-generated information. It is not impossible that financial information stored in a company's database may be continuously updated, audited, summarized, and filed with EDGAR.

\section{B. Continuous Auditing}

Auditing on reported information must be made on a continuous basis if financial information is continuously reported with the SEC. We can imagine that auditing process must be fast and still be reliable. There will be more emphasis on internal control for data processing than the EDP audits. For independent auditors, the issues of continuous auditing will be a big challenge because the existing standards and procedures for EDP auditing will not be adequate and will have to be changed to meet new needs. 


\section{Database Auditing}

One essential part of using EDGAR is the use of database technique. For auditors, database auditing has been a tremendous task due to the current limited auditing tools available for EDP auditing. Many problems that arise from database auditing are uniquely different from tape file-oriented EDP auditing (see Scott 1978). The problems compound further if the database structures are complex.

Existing generalized auditing software packages are not capable of handling complex data structure maintained in database. Today, auditors can utilize the existing auditing functions in the clients' databases to audit data stored on the databases. However, there are some problems in connection with using clients' DBMS (Data Base Management System) software. Firstly, the auditing functions available in the clients' databases may not be able to deal with all the auditors' work. Secondly, the auditors may have to depend on the client's assistant to understand the database structure and it's reliance with the clients' DBMS software. Too much dependence on the integrity of the DBMS software will affect the auditors' independence. Auditors should be more technically knowledgeable of database structures in order to retain the independent status.

\section{Efficient and Relevant Financial Analysis}

Continuous reporting may become available in the future with EDGAR. Timely information for investors will be available virtually instantaneously. Through EDGAR, investors or financial analysts can gain access to continuously available corporate financial information very quickly and conveniently (Goodman and Van Brunt, 1986; Jayson 1985; Van Brunt and Folbigg 1986). Investors may be able to access the data at home through microcomputers which are very cost-effective. Financial data can be analyzed using financial analysis software packages such as electronic spreadsheets. Users could prepare financial statements under a variety of accounting assumptions (Beaver 1984). Sounder financial and investing decisions can be made because data available is more up-to-date and relevant.

\section{E. Amount of Information Reported}

EDGAR not only offers a radical change in the way financial reporting is gathered, reported and disseminated but also in the essential character of the reporting. It has been a constant problem of financial reporting to decide the amount of information to be disclosed and the basis of reporting such information. Financial analysts and investors always complain that they favor more financial information being disclosed. However, it is one of the beliefs of the FASB that benefits of such disclosure should outweigh the cost of preparing them. With EDGAR, the efficiency of financial reporting can greatly reduce the cost of preparing such disclosure. It will not be a great surprise that more financial information will be available through EDGAR. On the other hand, when there is a steady flow of financial information available, the individual can also limit the kind and number of financial information he or she desires.

\section{F. References for CPAs}

When a CPA encounters any accounting problem or is reporting specifications while serving a client, the most common practice is to look into a similar company in that particular industry or to look at one that has a similar situation. Although a number of publications and on-line databases are now in the market, there is little doubt that EDGAR will become the dominant reference for CPAs when it becomes fully operational. All other sources contain only an extract of secondary information or a copy from either the original annual reports or the SEC filings. EDGAR is the primary source where companies will actually file their report with the SEC. The comparative advantage is obvious. Moreover, while other reference sources have to wait for such information before they can be transformed for users' needs, EDGAR is the original disseminating device. Thus, EDGAR will be more authoritative, timely and accurate as a reference source when compared with others. Auditors will need to be 
familiar with the EDGAR system in order to better fulfill their fiduciary duties.

\section{Conclusion}

The SEC's EDGAR system has been around for six years. Its development was the first step taken by the SEC to close the gap between information technology and financial reporting. There has been a growing acceptance of the system. As the system continues to mature, we can expect that it will become a major financial and accounting database for investors. The benefits of EDGAR are recognized by different groups of people involved, namely, the SEC, the filing companies and the investing public. In addition to these groups of people, the accounting professionals should also be concerned with the system. Accounting professionals should be aware of the future development of the system as its future implications which could change today's accounting practices and standards dramatically, especially in the reporting and auditing areas. These future implications may soon become a reality.

\section{References}

1. Arnold, Jerry L. and Michael A. Diamond, EDGAR: The SEC's Pilot Program and Its Impact, Financial Executives Reesearch Foundation, 1986, p. 11.

2. $\quad$ Beaver, William H., "Financial Reporting Needs More Than the Computer," Business Week, August 13, 1984 , p. 16.

3. $\quad$ Goodfellow, James L., "Financial Reporting at the Speed of Light," CA Magazine, August 1985, pp. 36-38.

4. Goodman, Amy L. and Roy T. Van Brunt, "The implications of EDGAR for Certified Public Accountants," CPA Journal, June 1986, p. 101.

5. $\quad$ Jayson, Susan, "Filing Into the Future With EDGAR," Management Accounting, June 1985, p. 23.

6. Liebtag, Bill, "Fraudulent Financial Reporting," Journal of Accountancy, August 1986, pp. 76-77.

7. $\quad$ Scott, Geeorge T., "Auditing the Data Base," CA Magazine, October 1978, pp. 52-59.

8. $\quad$ Simon, Ruth, "Wait and See," Forbes, February 11, 1985, p. 135.

9. $\quad$ Smith, Geoffrey, "Toward a More Perfect Market," Forbes, December 17, 1984, p. 73.

10. Van Brunt, Roy T. and Robert C. Folbigg, "EDGAR - Informing the Public," Corporate Accounting, Summer 1986, pp. $23-24$.

11. Yang, David C., "The Perceived Impact of Database Accounting," Proceedings of the Twenty Second Conference of the Western American Accounting Association, 1987, pp. 234-235. 